\title{
A new rapid, low-cost and GPS-centric unmanned aerial vehicle incorporating in-situ multispectral oil palm trees health detection.
}

\author{
Bryan D. See ${ }^{*}$, Shaiful J. Hashim ${ }^{1}$, Helmi Z. M. Shafri ${ }^{2}$, Syaril Azrad ${ }^{3}$, Mohd. Roshdi Hassan ${ }^{4}$ \\ ${ }^{1}$ Department of Computer \& Communication Systems Engineering, Faculty of Engineering, Universiti Putra \\ Malaysia, 43400 UPM- Serdang, Malaysia \\ ${ }^{2}$ Department of Civil Engineering, Faculty of Engineering, Universiti Putra Malaysia, 43400 UPM-Serdang, Malaysia \\ ${ }^{3}$ Department of Aerospace Engineering, Faculty of Engineering, Universiti Putra Malaysia, 43400 UPM-Serdang, \\ Malaysia \\ ${ }^{4}$ Department of Mechanical \& Manufacturing Engineering, Faculty of Engineering, Universiti Putra Malaysia, 43400 \\ UPM-Serdang, Malaysia
}

\begin{abstract}
Malaysia as the second largest producer of palm oil in the world has an industry that provides sizeable economic benefits for the country in term of healthy food and export opportunities. The greatest threat to oil palm (Elaeis guineensis) cultivation in Malaysia is from stem rot, caused by the white-rot fungus ganoderma boniness, which if neglected, may affect almost half a million hectares of oil palm farmland by the year 2020. This dreaded disease has no known effective cure and its spread can be curbed by early detection. It is inevitable that remote sensing techniques using multispectral or hyper spectral sensors be employed on unmanned aerial vehicles (UAV) to serve as an exploratory platform to identify the issues and scope involved for the synchronisation of different sensors, georeferencing the data acquired to avoid identification problems of tree identification in homogenous farmlands and the calculation of vegetation indices. The work is successful for its ability to screen each individual tree within almost three hectares of farmland within one minute of flight time, calculate the plant health indicator in real-time and dispense away with the cumbersome work routines to prepare for the acquisition of geolocation referential data mapping a homogenous farmland. Given the economic parameters, the enormous size of plantations, and the state of UAVs reliability, this research further give credence to the two-stage approach of using a lower cost multispectral imager to blanket-screen all trees individually and subsequently deploying a hyperspectral imager to confirm the health of suspect trees that were previously identified at the earlier stage. Post-processing of the data is still possible since the entire image is stored on the computing platform's external storage.
\end{abstract}

Keywords: UAV, Remote sensing, UAS, Multispectral, Real-time NDV I, Oil palm, Plant health, Raspberry Pi. Accepted on December 19, 2018

\section{Introduction}

Malaysia, as the second largest producer of palm oil in the world, has an industry that provides sizeable economic benefits for the country in term of healthy food and export opportunities (raw or processed). The yield of the oil palm plantation depends largely on the crop health and the corresponding market price depends heavily on the oil palm quality. Both yield and quality can be adversely affected by disease for example Ganoderma Basal Stem Rot (BSR) disease [1]. It was estimated that the total area affected by BSR in 2020 would be around 443,430 hectares or 65.6 million of oil palm trees [2]. In a study of 216 plantations, it was reported that up to $43.32 \%$ of the potential yields can be lost to BSR, if no treatment or control measures are applied [3].

Currently, there is no known effective cure for this Ganoderma disease [4]. There are a few biochemistry methods which are not practical for a typical oil palm plantation which advocate meticulous methods such as stem collection and laboratory works which are elaborate, laborious, time-consuming and costly [5]. It is inevitable BSR detection will turn to remote sensing technology. Hyperspectral Imaging (HSI) sensors possess a huge number of continuous spectral bands to record spectral responses of materials over specific continuous wavelength. On the other hand, Multispectral Imaging (MSI) sensors have smaller number of bands and their bands are normally broader than those of HSI. Generally, healthy vegetation covers signify high reflectance in near infrared and low reflectance in visible region of electromagnetic spectrum.

Hyperspectral applications on ganoderma detection were undertaken on various topics. Researchers using various vegetation indices (some modified) and red edge position techniques demonstrated that early detection of Ganoderma infection is feasible with accuracy rates ranging from $73 \%$ to $84 \%$ although more investigations are required to improve the accuracy. It is further noted that red edge position techniques using Lagrangian Interpolation Technique showed superior results over other vegetation indices [6].

In another study conducted in Indonesia [7] to detect the BSR disease, multispectral remote sensing techniques were used. Six 
vegetation indices, Atmospherically Resistant Vegetation Index (ARVI), Green Blue Normalized Difference Vegetation Index (GBNDVI), Green Normalized Difference Vegetation Index (GNDVI), NDVI, Soil Adjusted Vegetation Index (SAVI) and Simple Ratio (SR) were utilized to discriminate healthy palms from infected ones by space borne multispectral Quick Bird sensor. Among the numerous researchers that advocates for a more cost-effective alternative to the costly and cumbersome satellite sensing techniques for agriculture applications, the choice of an unmanned aerial vehicle (UAV) platform for carrying various sensor payloads becomes increasingly attractive [8]. The researchers are inclined to the usage of commercial-off-the-shelf (COTS) components plus opensource flight controller software [9,10]. Pixhawk [11] is a single-board system encompassing the flight management module and the input-output module. The operating system (OS) used is NuttX, a real-time open-source OS. The hardware design files and schematics are available online.

MAVLink or Micro Air Vehicle Link is a protocol for communicating with small unmanned vehicle. It is designed as a header-only message marshalling library. One of its main functions is to download telemetry data in real-time such as Global Positioning System (GPS) coordinates, vehicle airspeed, altitude and other data which are the primary raw information targets of this paper.

In one of the earliest work to test the suitability of using commercial cameras as MSI, [12] the authors concluded that the use of raw, unprocessed image data did not improve the results of image analyses. Furthermore, they contend that vignetting had a significant effect, especially for the modified camera, and normalized vegetation indices calculated with vignetting-corrected images were sufficient to correct for scene illumination conditions. The researchers work on the development of UAV-based sensing systems for precision agriculture. Their efforts to use the Red Green Blue Vegetation Index (RGBVI) and NDVI to meet their research objectives, provided insights into the effectiveness of using such vegetation indices [13].Though the mission objectives of this research are different, the materials used are similar to the ones used in this paper. The Raspberry Pi vision module is aimed to augment the flight control system, whilst in this paper, the vision module is meant for multispectral data acquisition [14].

\section{Methodology}

Due to budgetary constraints, a UAV quadcopter configuration was adopted by using the propulsion (motors, electronic speed controllers (ESC), propellers and battery from a semi-discarded Walkera Scout X4 quadcopter. Table 1 shows the complete list of major parts and components used in this quadcopter build while Figure 1 displays the complete configuration with all the functional communication interfaces.

Figure 2 shows a picture of the completed UAV. Its final weight was $2370 \mathrm{~g}$, slightly above the recommended maximum take-off weight by about $120 \mathrm{~g}$. Tuning of this quadcopter was done using the Auto-tune feature provided by the Pixhawk flight controller firmware. It was then manually tuned, after which the UAV was easy to fly, especially operating in autonomous mode.The multispectral data acquisition system was powered by two Raspberry Pi 3 which are linked together using a standard asynchronous link of 921600 bits per second. Both the Raspberry Pi's used the standard Raspbian Jessie operating system (a variant of Debian 8 Linux) together with the Python programming language.

Table 1. Components list (USD 1=MYR 4)

\begin{tabular}{|c|c|c|}
\hline Item & Description & Cost in USD \\
\hline 1 & $1 \times$ HobbyKing $X 580$ quadcopter frame & 35 \\
\hline 2 & 4 xWalkera Scout $X 4$ motors & 140 \\
\hline 3 & 4 xWalkera Scout X4 ESC & 104 \\
\hline 4 & $2 \times$ Walkera Scout $\mathrm{X} 4$ propellers $(\mathrm{CW}+\mathrm{CCW})$ pairs & 15 \\
\hline 5 & 1 x $6 \mathrm{~S} 22.2$ V 5400 mAh Lipo battery & 110 \\
\hline 6 & 1 x 3S 11.1 V 2200 mAh Lipo battery & 20 \\
\hline 7 & 2 x Raspberry Pi Model 3B & 70 \\
\hline 8 & 1 x Raspberry Pi RGB Camera v2.1 & 25 \\
\hline 9 & 1 x Raspberry Pi NolR Camera v2.1 & 25 \\
\hline 10 & $1 \times$ FrSky Taranis X9D transmitter with $\mathrm{X} 8 \mathrm{R}$ receiver & 240 \\
\hline 11 & Pixhawk 2.4.8 with $915 \mathrm{Mhz}$ telemetry pair & 100 \\
\hline 12 & 1 x Ublox M8N GPS/Compass & 40 \\
\hline 13 & $433 \mathrm{MHz}$ telemetry pair & 30 \\
\hline \multirow[t]{2}{*}{14} & Miscellaneous parts & 50 \\
\hline & Total & 1004 \\
\hline
\end{tabular}

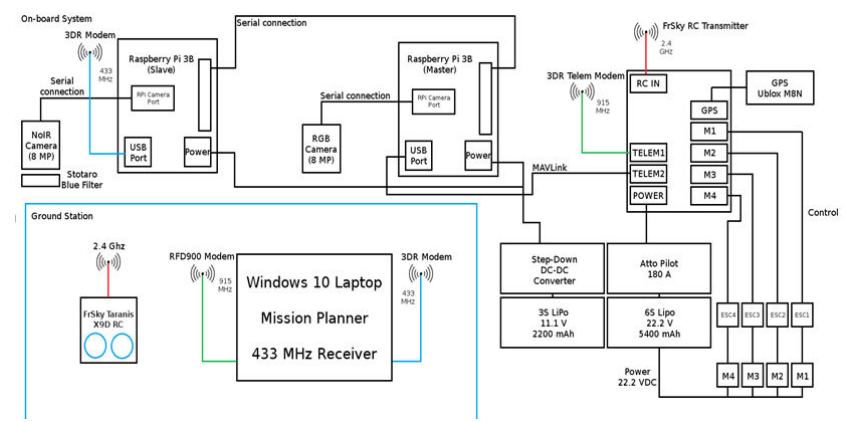

Figure 1. Overall configuration.

The first Raspberry Pi, termed the master, was equipped with a standard Raspberry Pi RGB 8- megapixel camera. It was linked to the Pixhawk flight controller via a serial link using the MAVlink protocol to receive in real-time, telemetry data such as GPS coordinates and altitude data.The second Raspberry Pi, termed the slave, was equipped with a 8 megapixel NoIR camera which admits near infrared (NIR) component. The lens was covered with the supplied blue filter (Roscolux 2007 Storaro Blue) to block out the red-component. Hence, to calculate the modified blue NDVI, the following formula was used:

$\mathrm{NDVIb}=(\mathrm{NDVI}-\mathrm{Blue}) /(\mathrm{NDVI}+\mathrm{Blue})$ 
Citation: See BD, Hashim SJ, Shafri HZ, et al. A new rapid, low-cost and GPS-centric unmanned aerial vehicle incorporating in-situ multispectral oil palm trees health detection. J Agric Sci Bot 2018;2(4):12-16.

After each NDVI image was generated, a simple notification completion message was sent to the Ground Control Station (GCS) via a radio link operating at $433 \mathrm{MHz}$.

Figure 3 shows the python scripts flowcharts for both the raspberry pi's. During the data acquisition stage, the UAV was flown at constant velocity of $5 \mathrm{~ms}^{-1}$ at various altitudes of 40 , 60 and 80 meters respectively. All flights were completely autonomous including take-offs and landings.

\section{Results and Discussion}

Given the raspberry pi camera field of view (FOV) angle is 62.20, Table 2 shows the calculated parameters pertaining to the three flights conducted. The assumptions used were a constant flight speed of $5 \mathrm{~ms}^{-1}$; the flight time of the battery used in the UAV is estimated to be between 15 and 20 minutes; and the image resolution used was $3280 \times 2464$ pixels i.e. 8 megapixels.

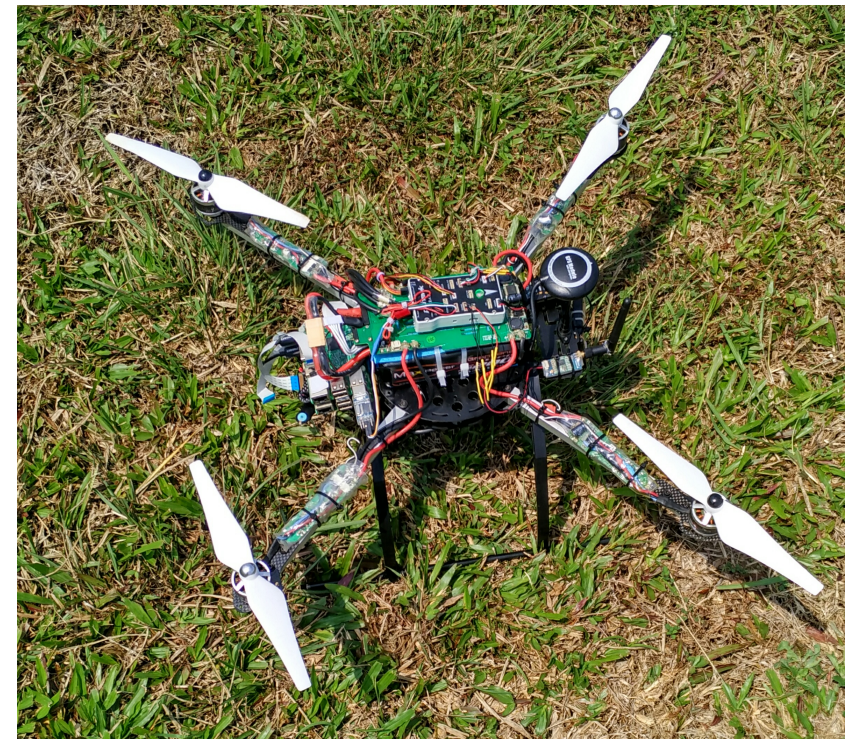

Figure 2. Complete UAV system.
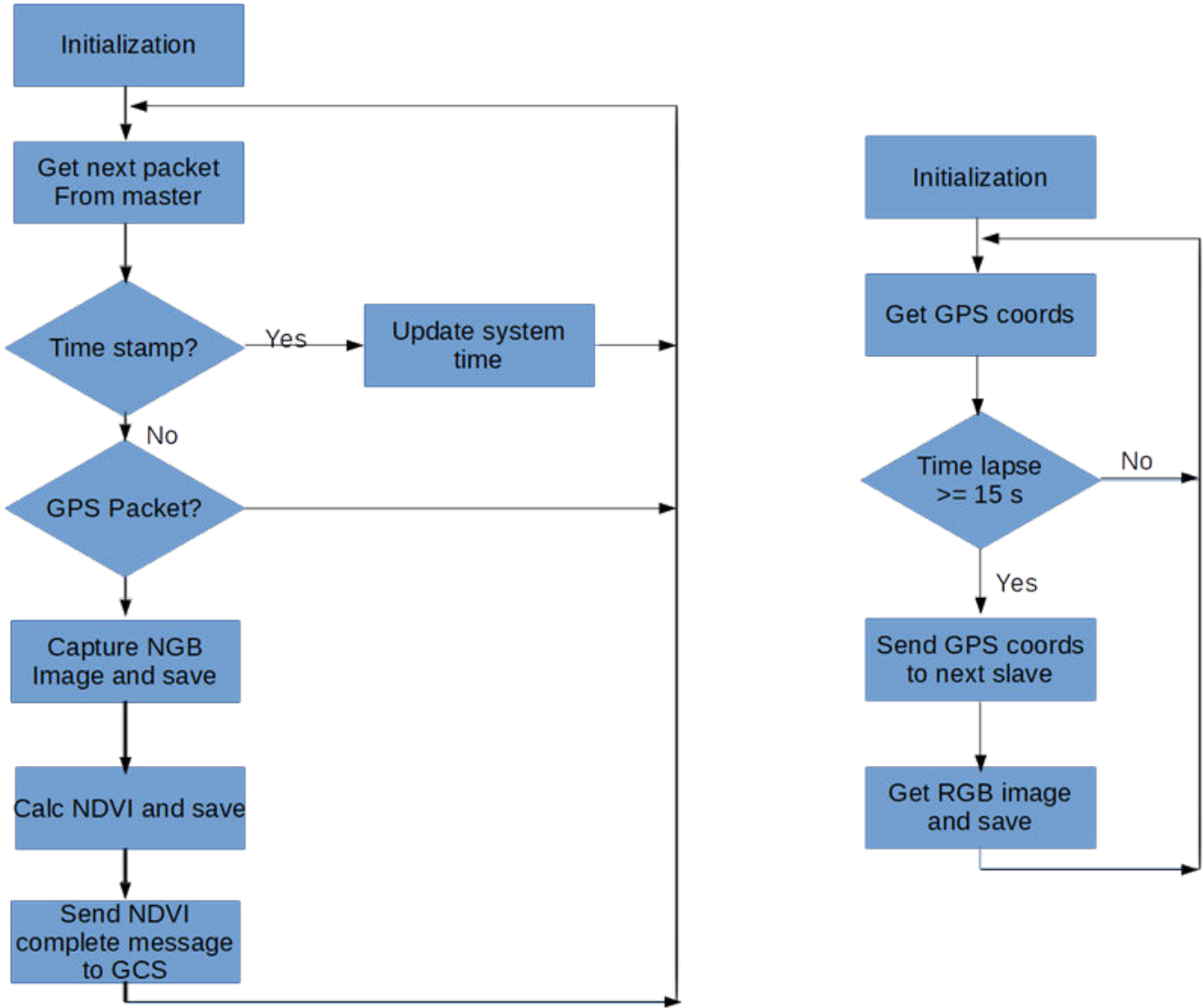

Figure 3. Flowcharts for master (left) and slave (right).

Table 2. Summary of flight statistics.

\begin{tabular}{|c|c|c|c|}
\hline Altitude (m) & 40 & 60 & 80 \\
\hline Image horizontal distance $(\mathrm{m})$ & 48.2 & 72.4 & 96.5 \\
\hline Image vertical distance $(\mathrm{m})$ & 36.2 & 54.3 & 72.4 \\
\hline
\end{tabular}




\begin{tabular}{|c|c|c|c|}
\hline Area covered (hectares) for one image & 0.174 & 0.393 & 0.698 \\
\hline Potential area covered in typical 15 minutes flight at $5 \mathrm{~m} / \mathrm{s}$ speed (hectares) & 10.5 & 23.6 & 41.9 \\
\hline Spectral resolution $(\mathrm{cm})$ & 1.47 & 2.21 & 2.94 \\
\hline
\end{tabular}

Figures 4 to 6 below show images acquired by the UAV at altitudes of $40 \mathrm{~m}, 60 \mathrm{~m}$ and $80 \mathrm{~m}$ respectively. All images have full resolution of 3280 bt 2464 pixels. Each figure consists of a RGB image that was acquired by the master Raspberry Pi, while the NGB image and the calculated NDVI image was provided using a false-color scale. All the NDVI images were generated in situ within a 12-14 seconds range on the slave Raspberry Pi.

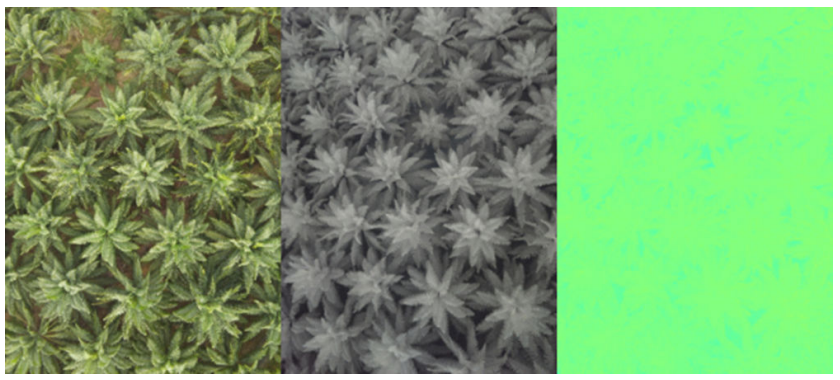

Figure 4. $R G B$ (left), NGB (middle), NDVI false color (right) for GPS N2.9882737O E101.7263544O altitude $40 \mathrm{~m}$.

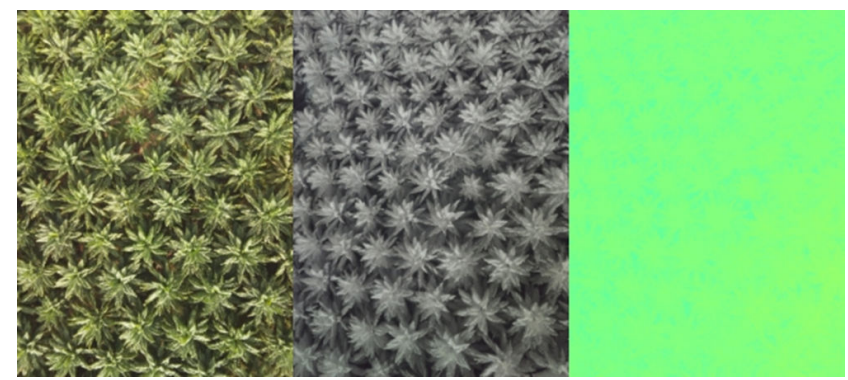

Figure 5. $R G B$ (left), NGB (middle), NDVI false color (right) for GPS N2.9882734O E101.726354O altitude $60 \mathrm{~m}$.

It is often desirable for a system to detect BSR or plant health would be for it to have some tree-crown delineating algorithm which is akin to tree-counting methodologies. A sample of the tree-crown delineating is shown at $80 \mathrm{~m}$ altitude in Figure 7 which was carried out as a post-analysis exercise. It can be deduced that this algorithm provided a fair amount of accuracy in identifying individual trees, although there is some degree of aggregation of trees which hamper the individual tree identification process.

\section{Cost Analysis}

The main component in the Mean Time between Failure (MTBF) calculations for an electric UAV is the bearings of the brushless direct current (BLDC) motor. The following bearing calculations are based on the standard ANSI-AFBMA Standard 9-1990 "Load Rating and Fatigue Life for Ball Bearing". Based on these calculations, the MTBF for the highly rated BLDC motors from Tiger Motor is 160 hours [15]. Based on a company's annual report, Boustead Plantations Bhd., which has over 65,000 hectares under oil palm cultivation, it revealed that its average cost of palm oil production increased to RM1,731 per metric ton (Boustead Plantations Bhd., 2017).

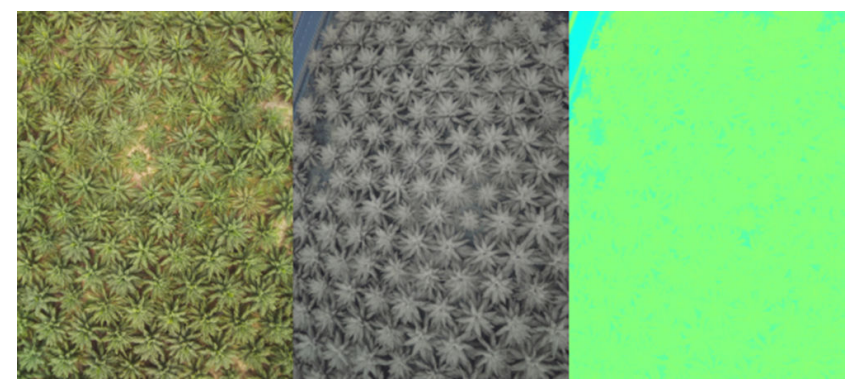

Figure 6. $R G B$ (left), $N G B$ (middle), NDVI false color (right) for GPS N2.988274O E101.7263549O altitude $80 \mathrm{~m}$.

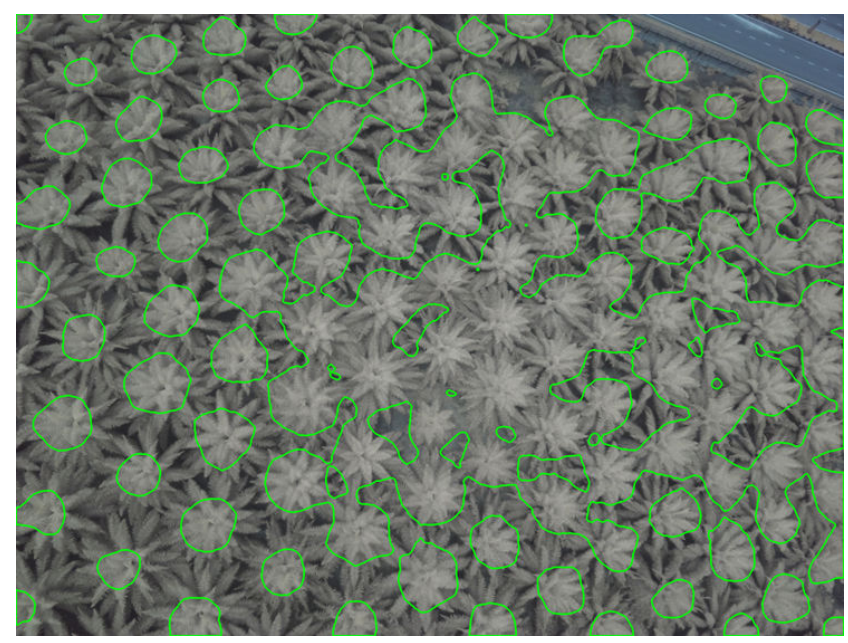

Figure 7. Post-analysis tree-crown delineating at $80 \mathrm{~m}$ altitude.

onsidering that a typical hectare produces about 5 tons of crude palm oil, the cost of production for a hectare of oil palm trees would be RM8,655 per year. It can be assumed that $1 \%$ of this amount can be afforded for a plant-health and/or disease detection service; this works out to be RM86.55 per hectare per year. From the empirical data collected and calculated in Table 1 , the area scanned in a 15-minute flight is 40 hectares, which means the area scanned in 1 hour will be 160 hectares. If we further assume that actual flight time is limited to 3 hours per day, the area that can be scanned in a day will be 480 hectares.

The quad copter used in this research has a replacement cost of USD1,004 (Table 1), excluding spares and shipping charges. Using the exchange rate at the time of writing, which is USD1=RM 4, this translated to a cost of RM 4,000. Assuming the UAV is completely written off after 100 hours of flight (considering assumption 1); this UAV would be able to scan 16,000 hectares $(160 \times 100)$ in its lifetime. Hence the cost of 
Citation: See BD, Hashim SJ, Shafri HZ, et al. A new rapid, low-cost and GPS-centric unmanned aerial vehicle incorporating in-situ multispectral oil palm trees health detection. J Agric Sci Bot 2018;2(4):12-16.

the UAV portion is RM0.25 (4000/16000) per hectare. A team of 3 persons is required to fly the UAV can be outsourced for RM1,500 per day in Malaysia, which will result in a cost of RM3.125 per hectare (1500/480). The labour cost and UAV amortization cost are the two main constituents, RM3.125 and RM0.25 respectively. Even if the UAV portion were to increase by a factor of 10 or 20 times, the total cost per scan would be less than RM10 per hectare. The obvious limitation to this scheme is that the recommended solution is for large oil palm plantations of 480 hectares and above. For smaller-sized farms, a slightly different approach will be required. Perhaps a more autonomous UAV platform with an operator-friendly user interface can mitigate the 3-man team requirements.

\section{Conclusion}

The work done for this thesis formed the initial exploratory platform for a potentially larger project for the early detection of Ganoderma boninense disease among Elaeis guineensis (oil palm) trees using remote sensing techniques. The work is successful for its ability to screen each individual tree within three hectares of farmland within one minute of flight time, calculate the plant health indicator in real-time and dispense away with the cumbersome work routines to prepare for the acquisition of geolocation referential data mapping a homogenous farmland. Given the economic parameters, the enormous size of plantations, and the state of UAVs reliability, this thesis further give credence to the two-stage approach of using a lower cost MSI to blanket-screen all trees individually and subsequently deploying a HSI to confirm the health of suspect trees that were previously identified at the initial stage. Post-processing of the data is still possible since the entire images are stored on the Raspberry Pi's micro SD card.

\section{References}

1. Ariffin D, Idris AS, Singh G. Status of Ganoderma in oil palm. Ganoderma diseases of perennial crops. J Flood, PD Bridge M. Holderness. 49-68

2. Roslan A, Idris AS. Economic impact of Ganoderma incidence on Malaysian oil palm plantation-a case study in Johor. Oil Palm Industry Economic J. 2012;12(1):24-30.

3. Assis K, Chong KP, Idris AS, et al. Economic loss due to ganoderma disease in oil palm. World Academy of Science, Engineering and Technology, International $\mathrm{J}$ Social, Behavioral, Educational, Economic, Business Industrial Engineering. 2016;10(2):631-5.

4. Lim KH, Lim SS, Parish F, et al. RSPO manual on best management practices (BMPs) for existing oil palm cultivation on peat. RSPO, Kuala Lumpur. 2012.

5. Sankaran S, Mishra A, Ehsani R, et al. A review of advanced techniques for detecting plant diseases. Comput Electron Agric. 2010;72(1):1-13.
6. Shafri HZ, Anuar MI, Saripan MI. Modified vegetation indices for Ganoderma disease detection in oil palm from field spectroradiometer data. J Appl Remote Sens. 2009;3(1):033556.

7. Santoso H, Gunawan T, Jatmiko RH, et al. Mapping and identifying basal stem rot disease in oil palms in North Sumatra with QuickBird imagery. Precision Agriculture, 2011;12(2):233-48.

8. Velasquez LC, Argueta J, Mazariegos K. Implementation of a low cost aerial vehicle for crop analysis in emerging countries. In Global Humanitarian Technology Conference (GHTC).2016;2016:21-27. IEEE.

9. Meier L, Tanskanen P, Fraundorfer F, et al. The PIXHAWK open-source computer vision framework for MAVs. The International Archives of the Photogrammetry, Remote Sensing and Spatial Information Sciences. 2012;38(1/C22): 13-18.

10. Yang Z, Lin F, Chen BM. Survey of autopilot for multirotor unmanned aerial vehicles. In Industrial Electronics Society, IECON 2016-42nd Annual Conference of the IEEE (pp. 6122-6127). IEEE. 2016.

11. Pohl C, Kanniah KD, Loong CK. Monitoring oil palm plantations in Malaysia. In Geoscience and Remote Sensing Symposium (IGARSS), 2016 IEEE International. 2016: 2556-9.

12. Lebourgeois V, Bégué A, Labbé S, et al. Can commercial digital cameras be used as multispectral sensors? A crop monitoring test. Sensors. 2008;8(11):7300-22.

13. Bareth G, Bolten A, Gnyp ML, et al. Comparison of uncalibrated RGBVI with spectrometer-based NDVI derived from UAV sensing systems on field scale. International Archives of the Photogrammetry, Remote Sensing \& Spatial Information Sciences. 2016; 837-43.

14. Choi H, Geeves M, Alsalam B, et al. Open source computer-vision based guidance system for UAVs on-board decision making. Aerospace Conference. 2016;1-5.

15. Quadquestions.com. T-Motor U7-420KV Brushless Motor. 2017.

\section{*Corresponding author:}

Bryan D. See

Department of Computer \& Communication Systems

Engineering

Faculty of Engineering

Universiti Putra Malaysia

43400 UPM-SerdangMalaysia

E-mail: bryan.see@live.com 